\title{
Report
}

\section{Regulation of Corepressor Function by Nuclear NADH}

\author{
Qinghong Zhang, ${ }^{1}$ David W. Piston, ${ }^{2}$ Richard H. Goodman ${ }^{1} *$ \\ ${ }^{1}$ Vollum Institute, Oregon Health Sciences University, 3181 SW Sam Jackson Park Road, Portland, OR 97201, USA. \\ ${ }^{2}$ Department of Molecular Physiology and Biophysics, Vanderbilt University, 702 Light Hall, Nashville, TN 37232, USA.
}

*To whom correspondence should be addressed. E-mail: goodmanr@ohsu.edu

The corepressor CtBP (carboxyl-terminal binding protein) is involved in transcriptional pathways important for development, cell cycle regulation, and transformation. We demonstrate that CtBP binding to cellular and viral transcriptional repressors is regulated by the nicotinamide adenine dinucleotides $\mathrm{NAD}^{+}$and NADH, with NADH being two-to-three orders of magnitude more effective. Levels of free nuclear nicotinamide adenine dinucleotides, determined using two-photon microscopy, correspond to the levels required for half-maximal CtBP binding and are considerably lower than those previously reported. Agents capable of increasing NADH levels stimulate CtBP binding to its partners in vivo and potentiate CtBP-mediated repression. We propose that this ability to detect changes in nuclear $\mathrm{NAD}^{+} / \mathrm{NADH}$ ratio allows $\mathrm{CtBP}$ to serve as a redox sensor for transcription.

CtBP was initially identified through its ability to interact with the carboxyl-terminus of adenovirus E1A. Mutation of the $\mathrm{CtBP}$ binding site in E1A decreases its transcriptional repression effects and increases its ability to direct cellular transformation $(1,2)$. CtBP also participates in the actions of cellular transcription factors involved in growth and differentiation, as demonstrated in Drosophila (3) and vertebrate systems (4). The recent demonstration that yeast Sir2 utilizes $\mathrm{NAD}^{+}$as a substrate (5-7) and the remarkable sequence conservation of $\mathrm{CtBP}$ with the dehydrogenases and reductases (2) (Fig. 1A), enzymes that utilize nicotinamide adenine dinucleotides as cofactors, led us to ask whether $\mathrm{CtBP}$ might similarly be regulated by $\mathrm{NAD}^{+}$or $\mathrm{NADH}$.

$\mathrm{CtBP}$ expressed in bacteria or isolated from HeLa cells was incubated with glycerophosphate, acetoacetate, pyruvate, lactate, acetate, formate, and ethanol in the presence of $\mathrm{NAD}^{+}$ or NADH under a variety of experimental conditions. No dehydrogenase or reductase activity was detected. We next tested whether CtBP was regulated in some other manner by $\mathrm{NAD}^{+}$or NADH. This hypothesis was suggested by the near perfect conservation of the $\mathrm{NAD}^{+} / \mathrm{NADH}$ binding signature near the middle of the CtBP sequence (Fig. 1B). One possibility was that $\mathrm{NAD}^{+} / \mathrm{NADH}$ could affect the ability of $\mathrm{CtBP}$ to interact with its partners. To test this hypothesis, we examined the interaction of bacterially-expressed CtBP with GST-E1A fusion proteins at different concentrations of $\mathrm{NAD}^{+} / \mathrm{NADH}$. To our surprise, $\mathrm{CtBP}$ binding was regulated dramatically, with NADH increasing the interaction at concentrations in the $\mathrm{nM}$ range (Fig. $1 \mathrm{C}$ ). $\mathrm{NAD}^{+}$also increased binding, but was 2 to 3 orders of magnitude less effective. NADP ${ }^{+}$, NADPH, and $\mathrm{FAD}^{+}$had little effect (Fig. 1D) (data not shown). $\mathrm{NAD}^{+} / \mathrm{NADH}$ similarly affected $\mathrm{CtBP}$ binding to a prototypical cellular repressor, ZEB (Fig. 1E), which is known to block transcription at least in part via CtBP interactions (8).

Knowing the physiological concentrations of free nuclear $\mathrm{NAD}^{+} / \mathrm{NADH}$ is critical for assessing whether these molecules regulate $\mathrm{CtBP}$ function in vivo. Using two-photon excitation microscopy, one can determine the concentration of NAD $(\mathrm{P}) \mathrm{H}$ in different cellular compartments (9). This was done in $\operatorname{Cos} 7$ cells by quantitative imaging of the total intensity and lifetime of NAD(P)H fluorescence. For these measurements, NADH and NADPH are indistinguishable, so we measure the sum of both molecules. Comparing the total intensity to a standard curve of free $\mathrm{NAD}(\mathrm{P}) \mathrm{H}$ in solution, we found that the nucleus contained $113 \mu \mathrm{M}$ NAD(P)H (Fig. 2A). Precise determination of the concentration is complicated by the fact that the fluorescence of free and bound forms of NAD $(\mathrm{P}) \mathrm{H}$ differs. Free NAD $(\mathrm{P}) \mathrm{H}$ has a considerably lower quantum efficiency than that bound to protein. Because the quantum efficiency is associated with the fluorescence lifetime, we can determine the fraction of bound $\mathrm{NAD}(\mathrm{P}) \mathrm{H}$ by fluorescence lifetime imaging (10). The fluorescence lifetime image was homogeneous across all subcellular compartments (Fig. 2B) with a value of $3.41 \mathrm{nsec}$ $(\mathrm{n}=6$ cells), as compared to $0.45 \mathrm{nsec}$ for free NAD(P)H. This indicates that the vast majority of NAD(P)H is bound and that our estimate of $113 \mu \mathrm{M}$ is $\sim 7.5$-fold too high (ratio of 3.41 to 0.45$)$. The corrected nuclear $\mathrm{NAD}(\mathrm{P}) \mathrm{H}$ concentration is thus $\sim 15 \mu \mathrm{M}$. To quantitate the amount of free $\mathrm{NAD}(\mathrm{P}) \mathrm{H}$, we performed a multifrequency experiment with phase modulations at 80,160 , and $240 \mathrm{MHz}$ and fit multiple exponential decays to the fluorescence lifetimes. One lifetime component was fixed at $0.451 \mathrm{nsec}$ (the lifetime for free $\mathrm{NAD}(\mathrm{P}) \mathrm{H})$, and the other was allowed to vary with the nonlinear least squares fit. According to this fit, the fraction of fluorescence associated with the free component was $4.4 \pm$ $2.7 \%$. Thus, the upper limit of free $\mathrm{NAD}(\mathrm{P}) \mathrm{H}$ is $660 \mathrm{nM}$. If we assume that NADPH/NADH ratio is approximately 4 (11), the concentration of free NADH in the nucleus is $\sim 130$ $\mathrm{nM}$ (12), well within the range required for stimulating the E1A:CtBP interaction.

Because $\mathrm{NAD}^{+} / \mathrm{NADH}$ affected $\mathrm{CtBP}$ binding to multiple transcriptional repressors, we speculated that these cofactors most likely functioned by altering CtBP structure. Support for this idea was obtained from limited proteolysis experiments. In the absence of $\mathrm{NAD}^{+} / \mathrm{NADH}$, trypsin treatment releases a $10 \mathrm{kDa}$ fragment from the $\mathrm{CtBP}$ amino-terminus, resulting in the $30 \mathrm{kDa}$ fragment visualized in Fig. 3A. This fragment is not generated if CtBP is incubated with nicotinamide adenine dinucleotides. To confirm these findings, we examined the binding of $\mathrm{CtBP}$ containing a mutation in the $\mathrm{NAD}^{+} / \mathrm{NADH}$ interaction site (13). Although the basal interactions between E1A and the CtBP mutant were maintained, the stimulation by $\mathrm{NAD}^{+} / \mathrm{NADH}$ was lost (Fig. 3B). Additionally, 
$\mathrm{NAD}^{+} / \mathrm{NADH}$ did not protect the CtBP mutant from trypsin digestion (12). We conclude that $\mathrm{CtBP}$ binding to $\mathrm{E} 1 \mathrm{~A}$, and presumably other repressors, is regulated by $\mathrm{NAD}^{+} / \mathrm{NADH}$ and that $\mathrm{NADH}$ is far more effective in regulating binding.

One model consistent with our observations is that CtBP evolved from the dehydrogenases and reductases in a manner that resulted in loss of enzymatic activity but retention of the capacity to be regulated by $\mathrm{NAD}^{+} / \mathrm{NADH}$. To test this hypothesis, we asked whether the association of E1A and $\mathrm{CtBP}$ could be regulated by agents that perturb cellular redox state. E1A and FLAG-tagged CtBP were cotransfected into Cos7 cells, which were subsequently treated with $200 \mu \mathrm{M}$ $\mathrm{CoCl}_{2}, 10 \mathrm{mM} \mathrm{Na}$ azide, or hypoxia $\left(1 \% \mathrm{O}_{2}\right)$. Complexes were isolated using an anti-FLAG antibody, and Western blots were probed with antibodies against E1A and CtBP. The effects of these treatments on free nuclear $\mathrm{NAD}^{+} / \mathrm{NADH}$ cannot be measured directly, but an estimate of their effects on the free cytoplasmic pools can be derived from the [pyruvate]/[lactate] ratio $(12,14)$. Assuming no barrier to the diffusion of free NAD cofactors between the cytoplasmic and nuclear compartments, these measurements should reflect the free nuclear $\mathrm{NAD}^{+} / \mathrm{NADH}$ ratios. Each of the treatments decreased the free cytoplasmic $\mathrm{NAD}^{+} / \mathrm{NADH}$ ratio (Fig. $4 \mathrm{~A}$ ), probably via an increase in free NADH. Moreover, each treatment also increased the association of FLAG-CtBP with E1A (Fig. 4B). Absolute levels of E1A and CtBP were not affected.

To confirm that perturbations in $\mathrm{NAD}^{+} / \mathrm{NADH}$ ratios affected CtBP interactions in a manner that could regulate transcription, we performed mammalian two-hybrid assays. Cos7 cells transfected with VP16CtBP and GalZEB, a fusion gene containing the DNA binding domain of Gal4 fused to the CtBP binding domain of ZEB (8), were treated $8 \mathrm{hrs}$ after transfection with $200 \mu \mathrm{M} \mathrm{CoCl}_{2}$. ZEB:CtBP interactions were significantly increased by $\mathrm{CoCl}_{2}$ treatment (Fig. 4C). Western blots showed that the wild type and mutated VP16CtBP proteins were expressed at similar levels. Mutation of the $\mathrm{NAD}^{+} / \mathrm{NADH}$ binding site in $\mathrm{CtBP}$ virtually eliminated reporter activity, indicating that even the basal levels of nuclear $\mathrm{NAD}^{+} / \mathrm{NADH}$ can stimulate the ZEB-CtBP interaction. No interaction was detected when ZEB proteins containing mutated $\mathrm{CtBP}$ binding sites were used and no increase in activity was seen in control interactions (i.e., CREB:CBP) (12).

To test whether this pathway affects repression of a naturally-occuring promoter, we cotransfected Cos7 cells with an E-cadherin reporter gene and truncated ZEB constructs containing an E-box binding domain and wild type or mutated CtBP-binding motifs (15). As reported previously, $\mathrm{ZEB}$ repressed the E-cadherin promoter in a manner that depended on the CtBP interaction sites (Fig. 4D). Treatment with $\mathrm{CoCl}_{2}$ or hypoxia significantly enhanced the level of $\mathrm{CtBP}$-mediated repression. Neither $\mathrm{CoCl}_{2}$ nor hypoxia affected expression of the E-cadherin promoter in the presence of a ZEB mutant incapable of binding CtBP (data not shown).

The current study suggests that the transcriptional corepressor $\mathrm{CtBP}$ is regulated through binding of nicotinamide adenine dinucleotides. The concentration of $\mathrm{NAD}^{+} / \mathrm{NADH}$ required to stimulate the E1A-CtBP interaction in vitro is surprisingly low and reflects the low levels of nicotinamide adenine dinucleotides found in the nucleus (Fig. 2) (9). NAD ${ }^{+} / \mathrm{NADH}$ should readily pass through nuclear pores, suggesting that cellular perturbations that affect free cytoplasmic levels should also cause changes in the nuclear compartment. Levels of free nuclear nicotinamide adenine dinucleotides had not previously been determined, however. The Km of Hst2, a cytoplasmic Sir2-like histone deacetylase, for $\mathrm{NAD}^{+}$is $70 \mu \mathrm{M}(7)$, approximately the level required for half-maximal stimulation of CtBP-E1A binding (see Fig. 1D). Assuming that this value reflects the concentration of free $\mathrm{NAD}^{+}$and that the free $\mathrm{NAD}^{+} / \mathrm{NADH}$ ratio is 644 (see Fig. 4A), we estimate that the free NADH concentration is about $110 \mathrm{nM}$. Free nuclear NADH levels measured using twophoton microscopy confirm this estimation. Because free $\mathrm{NAD}^{+}$levels greatly exceed those of NADH, large changes in the $\mathrm{NAD}^{+} / \mathrm{NADH}$ ratio do not require correspondingly large changes in free $\mathrm{NAD}^{+}$. Thus, changes in nuclear redox could be manifested primarily through NADH, which is consistent with the higher sensitivity of $\mathrm{CtBP}$ to $\mathrm{NADH}$ than $\mathrm{NAD}^{+}$. Of interest, Rutter et al. (16) recently reported that binding of the transcription factor NPAS2 to DNA is also regulated by the redox state of NAD cofactors. The concentration of NADH and NADPH required in that study for half-maximal binding was approximately $10 \mathrm{mM}$, however, five orders of magnitude higher than the free nuclear concentrations that we have determined. Whether NPAS2 is sensitive to physiologically relevant levels of nicotinamide adenine dinucleotides thus remains to be determined. Large changes in cellular redox state occur at birth (17), in response to ethanol (18), and in certain metabolic abnormalities such as diabetes (14). Thus, the mechanism described in this report could influence multiple transcriptional repressor pathways. The best-characterized target promoter for $\mathrm{CtBP}$ in mammalian cells is probably the E-cadherin gene $(15,19)$ and loss of E-cadherin expression in tumors correlates with metastasis, invasion, and poor clinical prognosis $(20,21)$. Our studies indicate that $\mathrm{CtBP}$-mediated repression of the $\mathrm{E}$ cadherin promoter is enhanced by hypoxia. Because tumor cells are frequently hypoxic and thereby would be expected to have an increased NADH/NAD ${ }^{+}$ratio, we predict that the concomitant increase of ZEB-CtBP binding may contribute to tumor invasiveness.

\section{References and notes}

1. J. M. Boyd et al., EMBO J. 12, 469 (1993).

2. U. Schaeper et al., Proc. Natl. Acad. Sci. U.S.A. 92, 10467 (1995).

3. M. Mannervik, Y. Nibu, H. Zhang, M. Levine, Science 284, 606 (1999).

4. J. Turner, M. Crossley, Bioessays 23, 683 (2001).

5. S. Imai, C. M. Armstrong, M. Kaeberlein, L. Guarente, Nature 403, 795 (2000).

6. J. Landry et al., Proc. Natl. Acad. Sci. U.S.A. 97, 5807 (2000).

7. K. G. Tanner, J. Landry, R. Sternglanz, J. M. Denu, Proc. Natl. Acad. Sci. U.S.A. 97, 14178 (2000).

8. A. A. Postigo, D. C. Dean, Proc. Natl. Acad. Sci. U.S.A. 96, 6683 (1999).

9. G. H. Patterson, S. M. Knobel, P. Arkhammar, O. Thastrup, D. W. Piston, Proc. Natl. Acad. Sci. U.S.A. 97, 5203 (2000).

10. D. W. Piston, D. R. Sandison, W. W. Webb, Proc. SPIE 1640, 379 (1992)

11. K. Shigemori et al., Am. J. Physiol. 270, L803 (1996).

12. Supplementary data are available on Science Online at www.sciencemag.org/cgi/content/full/1069300/DC1.

13. M. Rescigno, R. N. Perham, Biochemistry 33, 5721 (1994). 
14. D. H. Williamson, P. Lund, H. A. Krebs, Biochem. J. 103, 514 (1967).

15. M. L. Grooteclaes, S. M. Frisch, Oncogene 19, 3823 (2000).

16. J. Rutter, M. Reick, L. C. Wu, S. L. McKnight, Science 293, 510 (2001).

17. H. Philippidis, F. J. Ballard, Biochem. J. 113, 651 (1969).

18. M. Stubbs, R. L. Veech, H. A. Krebs, Biochem. J. 126, 59 (1972).

19. J. Comijn et al., Mol. Cell 7, 1267 (2001).

20. S. Meiners, V. Brinkmann, H. Naundorf, W. Birchmeier, Oncogene 16, 9 (1998).

21. A. K. Perl, P. Wilgenbus, U. Dahl, H. Semb, G. Christofori, Nature 392, 190 (1998).

22. T. French, P. T. So, C. Y. Dong, K. M. Berland, E. Gratton, Methods Cell Biol. 56, 277 (1998).

23. We thank S. Frisch and D. Dean for reagents, H. Yao, C. Fjeld, N. Parry, R. Veech, and R. Hanson for discussion and assistance. Supported by grants from the NIH and NSF.

26 December 2001; accepted 2 February 2002

Published online 14 February 2002; 10.1126/science. 1069300

Include this information when citing this paper.

Fig. 1. $\mathrm{NAD}^{+} / \mathrm{NADH}$ regulates $\mathrm{CtBP}$ binding. (A) Alignment of bacterial phosphoglycerate dehydrogenase and human CtBP. (B) Alignment of putative $\mathrm{NAD}^{+} / \mathrm{NADH}$ binding sites. Gly residues important for binding are indicated. (C) Binding of recombinant CtBP to GST-E1A at various concentrations of NAD ${ }^{+}$and $\mathrm{NADH}$. Glutathione beads were coated with GST-E1ACter (carboxyl-terminal 67 amino acids of E1A) and bound CtBP was quantified by Western blotting. (D) Relative interactions as a function of nicotinamide adenine dinucleotide concentration (log scale). (E) Binding of recombinant $\mathrm{CtBP}$ to $\mathrm{GST}-\mathrm{ZEB}_{595-720}$ at various concentrations of $\mathrm{NAD}^{+}$and $\mathrm{NADH}$.

Fig. 2. Determination of nuclear NADH concentration. (A) Two-photon excitation imaging of NAD(P)H shows the autofluorescence intensity from a typical $\operatorname{Cos} 7$ cell. The color bar indicates the range of $\mathrm{NAD}(\mathrm{P}) \mathrm{H}$ from 0 (dark red) to 1 $\mathrm{mM}$ (white). Note paucity of signal in the nucleus $(\mathrm{N})$. (B) Lifetime image from the same cell, acquired by phasemodulation techniques using the instrument described by French et al. (22). The same color bar is used, but for this figure the range represents lifetime from 0 to $10 \mathrm{nsec}$ for each pixel in the cell rather than intensity. The average lifetime in the nucleus as well as other subcellular compartments was $3.41 \mathrm{nsec}$.

Fig. 3. $\mathrm{NAD}^{+} / \mathrm{NADH}$ induces a conformational change in CtBP. (A) Limited proteolytic digestion of CtBP without (control) or with $100 \mu \mathrm{M} \mathrm{NAD}^{+}$or $100 \mu \mathrm{M}$ NADH using various doses of trypsin. Western blots were developed using antibodies to CtBP- or a carboxyl-terminal His-epitope (Qiagen), as indicated. The $30 \mathrm{kDa}$ fragment (indicated by arrows) generated in the absence of $\mathrm{NAD}^{+} / \mathrm{NADH}$ results from the loss of a $10 \mathrm{kDa}$ amino-terminal fragment. (B) The $\mathrm{NAD}^{+} / \mathrm{NADH}$ binding site in $\mathrm{CtBP}$ is required for the stimulated interaction. CtBP proteins, wild type (WT) or mutated (G183A), were tested for their ability to bind to E1A in the absence (con) or presence of increasing concentrations $(100 \mathrm{nM}-100 \mu \mathrm{M})$ of $\mathrm{NAD}^{+}$or NADH by GST pull-down assays.
Fig. 4. Redox state regulates CtBP interaction in vivo. (A) Effects of various treatments on the free cytoplasmic $\mathrm{NAD}^{+} / \mathrm{NADH}$ ratio. Cells were treated with $200 \mu \mathrm{M} \mathrm{CoCl}_{2}$, $10 \mathrm{mM}$ azide, or exposed to hypoxia for $16 \mathrm{hr}$. Cellular lactate and pyruvate were determined and the free cytoplasmic $\mathrm{NAD}^{+} / \mathrm{NADH}$ ratio was calculated as described by Williamson et al. (14). (B) $\mathrm{CoCl}_{2}$, azide, or hypoxia (1\% $\mathrm{O}_{2}$ ) increase the amount of E1A associated with CtBP. E1A and FLAG-tagged CtBP were cotransfected into Cos7 cells, complexes were isolated using anti-FLAG $\mathrm{M}_{2}$ matrix (Sigma), and Western blots were probed with antibodies against E1A (M73, Santa Cruz) and CtBP. Cells were treated with $200 \mu \mathrm{M} \mathrm{CoCl}{ }_{2}$ or $10 \mathrm{mM}$ azide for $1 \mathrm{hr}$, or exposed to hypoxia for $3 \mathrm{hr}$. Input panels show that these treatments did not change the levels of $\mathrm{CtBP}$ and $\mathrm{E} 1 \mathrm{~A}$. (C) $\mathrm{CoCl}_{2}$ increases the interaction of $\mathrm{ZEB}$ and $\mathrm{CtBP}$ in a mammalian two-hybrid assay. $\operatorname{Cos} 7$ cells were cotransfected with pairs of interacting components in the presence of a $5 \times$ Gal-E1b-luc reporter. The ZEB component is fused to the Gal DNA binding domain and the CtBP component to VP16. G183A mutation ablates the $\mathrm{NADH} / \mathrm{NAD}^{+}$binding site in $\mathrm{CtBP}$. ZEBmt represents $\mathrm{ZEB}_{700-776}$ with all three CtBP-binding sites mutated (8). (D) $\mathrm{CoCl}_{2}$ and hypoxia enhance $\mathrm{CtBP}$-mediated repression. Cos7 cells were cotransfected with an E-cadherin reporter gene and truncated ZEB constructs containing an E-box binding domain and wild type or mutated CtBP-binding motifs. Cells were treated with $200 \mu \mathrm{M} \mathrm{CoCl}{ }_{2}$ or exposed to hypoxia for 16 hr. 
a. bacterial PGDH MVKILVTDPLHED-..........-AIKILEEVGEVEVATGLTKEELLEKIKDADV-LV hCtBP1 VRPPIMNGPLHPRPLVALLDGRDCTVEMPILKDVATVAFCDAQSTQEIHEKVLNEAVGAL

bacterial PGDH VRSGTKVTRDVIEKAEKLKVIGRAGVGVDNIDVEAATEKGI IVVNAPDASSISVAELTMG hCtBP1 MYHTITLTREDLEKFKALRI IVRIGSGFDNIDIKSAGDLGIAVCNVPAASVEETADSTLC

bacterial PGDH LMLAAARNIPQATASLKRG--.---- EWDRKRFKGIELYGKTLGVIGLGRIGQDVVKRAK hCtBP1 HILNLYRRATWLHQALREGTRVQSVEQIREVASGAARIRGETLGI I GLGRVGQA VALRAK

bacterial PGDH AFGMNIIGYDPYIPKEVAESMGVELVDDINELCKRADFITLHVPLTPKTRHIIGREOIAL hCtBP1 AFGFNVLFYDPYLSDGVERALGLQRVSTLQDLLFHSDCVTLHCGLNEHNHHLINDFTVKQ

bacterial PGDH MKKNAIIVNCARGGLIDEKALYEALKEGKIRAAALDVFEEEPP - - KDNPLLTLDNVIGTP hCtBP1 MRQGAFLVNTARGGLVDEKALAQALKEGRIRGAALDVHESEPFSFSQGPLKDAPNLICTP

bacterial PGDH HQGASTEEAQKAAGTIVAEQIKKVLRGELAENVVNMPNI PQEKLGKLKPYMLLAEMLGNI CtBP1 HAAWYSEQASIEMREEAAREIRRAITGRIPDSLKNCVNK - - - - -DHLTAATHWASMDPAV

bacterial PGDH VMOVLDGSVNRVELIYSGELAKEKTDLIKRAFLKGLLSPILLAGINLVNAPIIAKNRNIN hCtBP1 VHPELNGAAYRYP--.--PG--.----VVGVAPTGIPAAVEGIVPSAMSLSHGLP

b. 141 TL $\dot{G} V I \dot{G} L \dot{G} R I G Q Q$ bacterial PGDH 176 TLGI IGLGRVGQA hCtBP1

d.

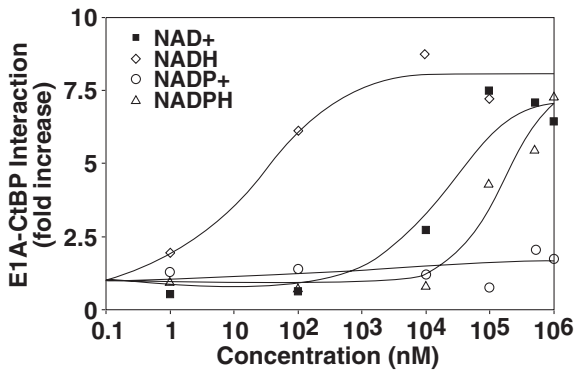

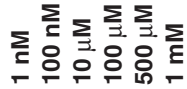
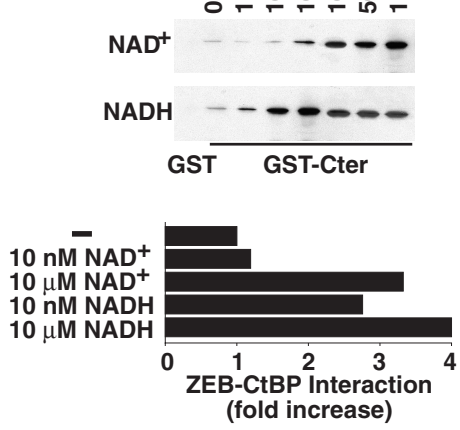


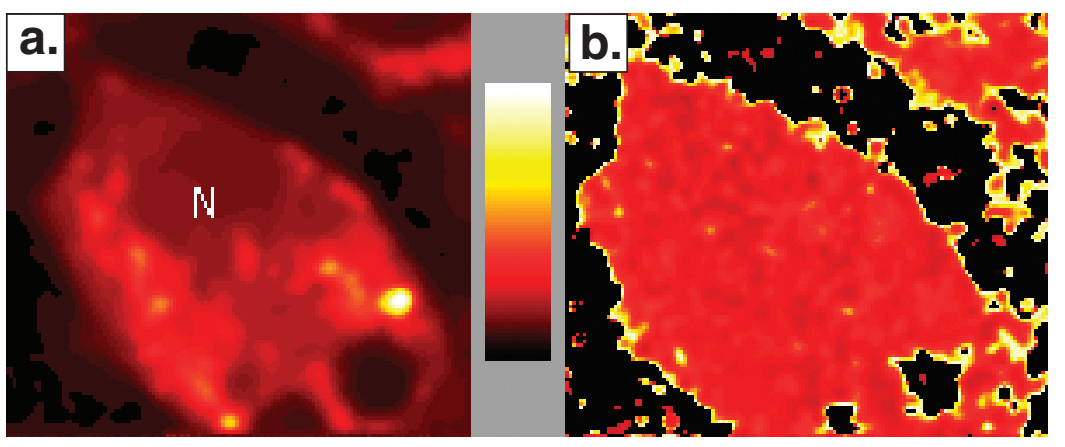


a.

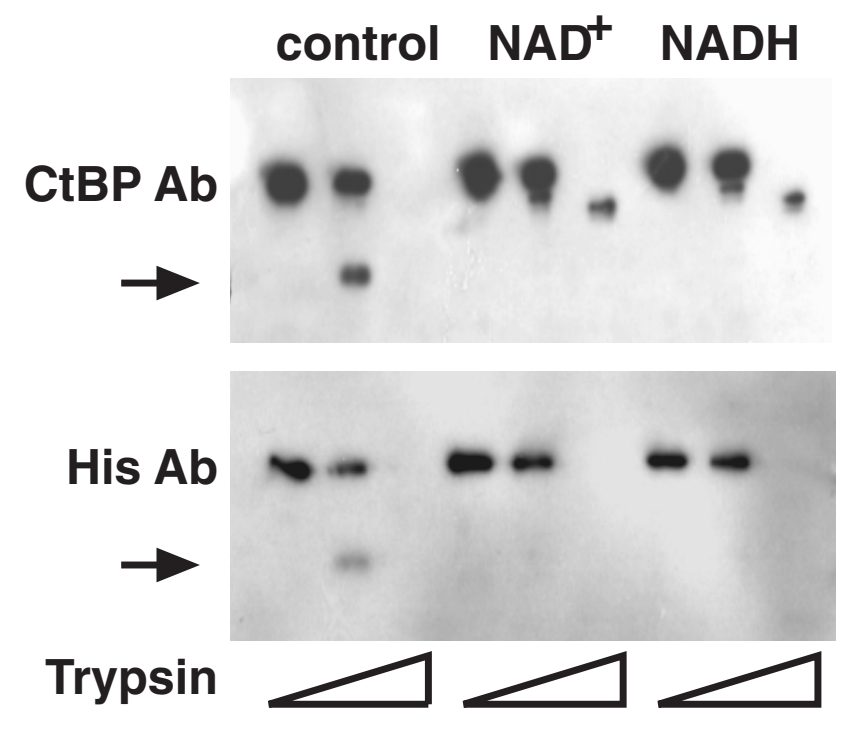

b.

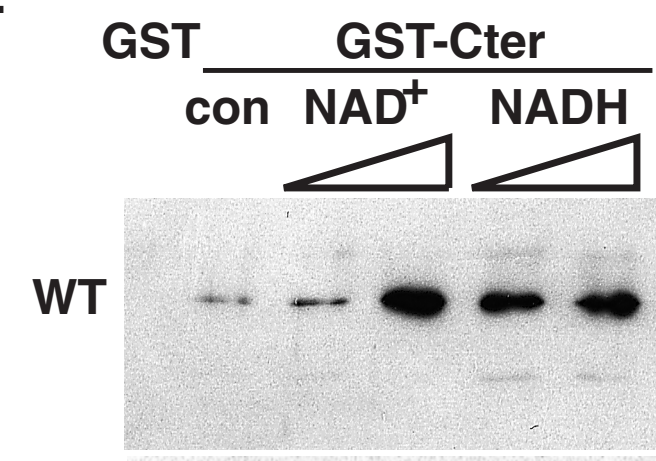

G183A 
a.

Free cytoplasmic

[NAD $\left.{ }^{+}\right]:[\mathrm{NADH}]$ ratio

control 644

$\mathrm{CoCl}_{2} \quad 413$

$\mathrm{NaN}_{3} \quad 314$

hypoxia 192

b.

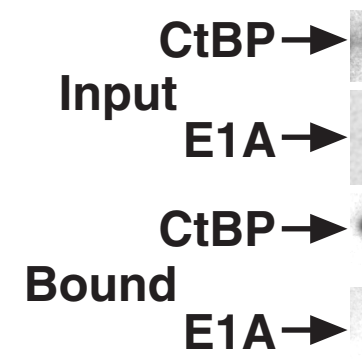

c.

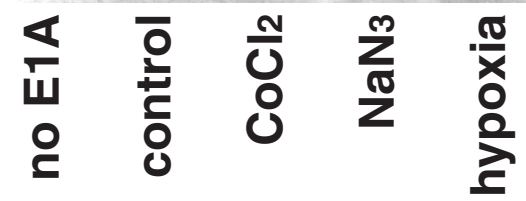

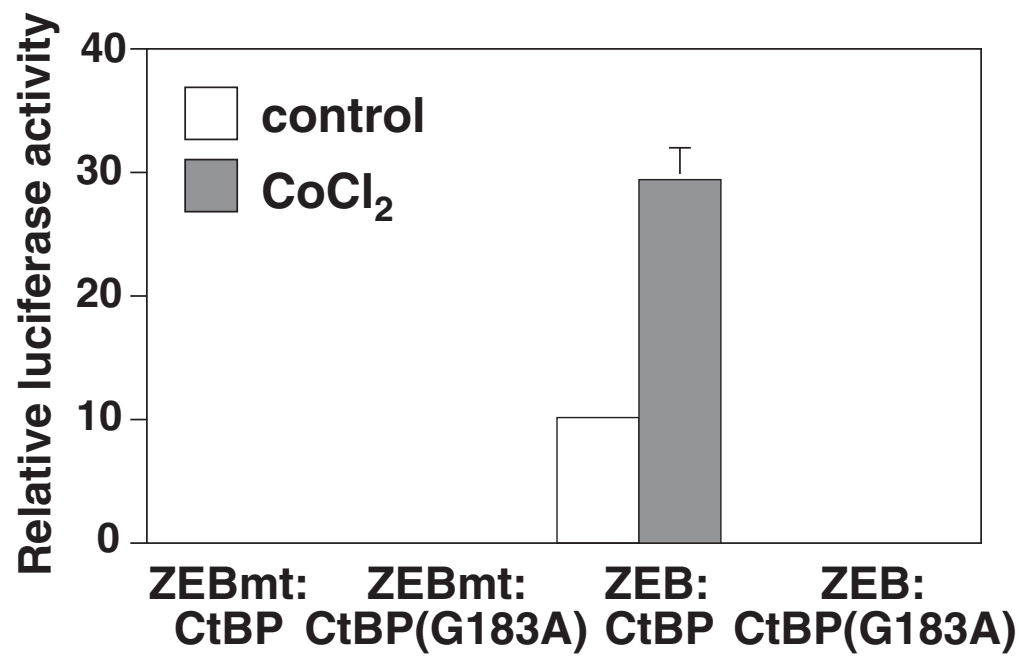

d.

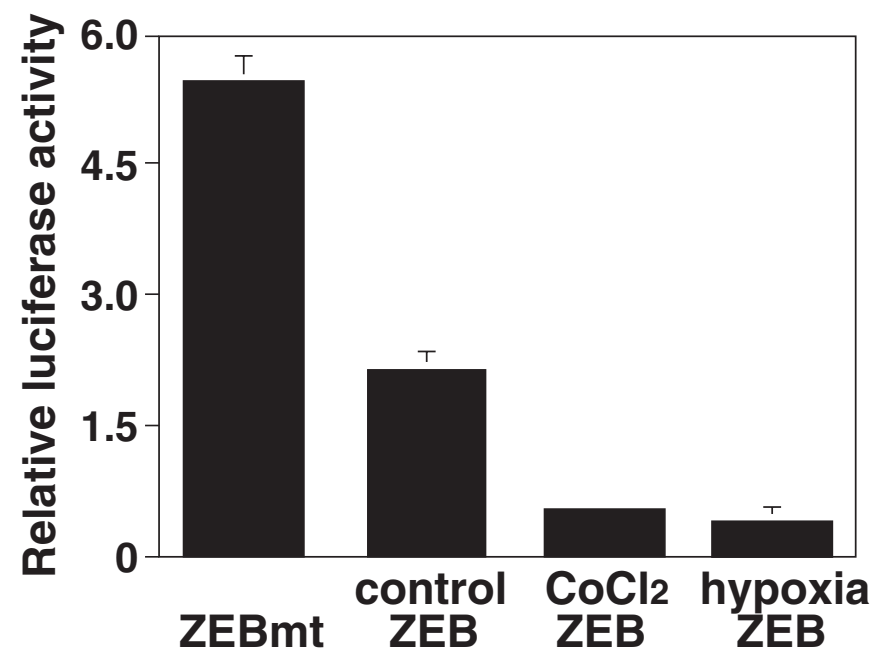

